Sains Malaysiana 47(7)(2018): 1473-1482

http://dx.doi.org/10.17576/jsm-2018-4707-15

\title{
Interaction Study of Binary Solvent Systems Ionic Liquid and Deep Eutectic Solvent with Rotenone
}

(Kajian Interaksi Sistem Pelarut Binari Cecair Ionik dan Pelarut Eutektik Dalam dengan Rotenon)

\author{
ZETTY SHAFIQA OTHMAN, MAMORU KOKETSU, NURUl HudA ABD KARIM, \\ SAIFUL IRWAN ZUBAIRI \& NUR HASYAREEDA HASSAN*
}

\begin{abstract}
[BMIM]OTf and alcohol-based DES combination with a selected organic solvent (acetone and acetonitrile) have been proven to efficiently extracting rotenone (isoflavonoid biopesticide) compound compared to individual organic solvents. Their efficiency builds up interest to study the solvent-solute interaction that occurs between both selected solvent systems with rotenone. The interaction study was analyzed using FTIR, 1D-NMR and 2D-NMR (NOESY, HMBC). Correlation portrayed by NOESY and HMBC of [BMIM]OTf - standard rotenone mixture predicted probable hydrogen bonding between the oxygen of rotenone with acidic proton C2-H of [BMIM]OTf. While for the alcohol-based DESrotenone mixture, the correlation shows probable interaction to occur between methyl and methoxy group rotenone with the hydroxyl group of 1,4-butanediol. In conclusion, potential hydrogen bonding that occurs between solvent and solute aid towards the solvent efficiency in extracting rotenone compound while emphasizing on the low cost and green mediated solvent systems.
\end{abstract}

Keywords: Biopesticide; deep eutectic solvent; extraction; ionic liquid; solvent-solute interaction

ABSTRAK

Gabungan [BMIM]OTf dan DES berasaskan alkohol dengan pelarut organik terpilih (aseton dan asetonitril) terbukti cekap dalam mengekstrak sebatian rotenon (biopestisid isoflavonoid) berbanding pelarut organik. Kecekapan tersebut menarik minat untuk mengkaji interaksi pelarut -bahan larut yang berlaku antara kedua-dua sistem pelarut terpilih dengan rotenon. Kajian interaksi dikaji menggunakan FTIR, ID-NMR dan 2D- NMR (NOESY, HMBC). Korelasi yang digambarkan oleh NOESY dan HMBC bagi campuran [BMIM]OTf - rotenon piawai menunjukkan potensi ikatan hidrogen antara oksigen rotenon dengan proton berasid C2-H [BMIM]OTf. Bagi campuran DES berasaskan alkohol-rotenon, korelasi menunjukkan interaksi berpotensi berlaku antara metil dan kumpulan metoksi rotenon dengan kumpulan hidroksil 1,4-butanediol. Kesimpulannya, potensi ikatan hidrogen yang wujud antara pelarut dan bahan larut membantu ke arah kecekapan pelarut dalam pengekstrakan sebatian rotenon selain faktor kos yang rendah dan sistem pelarut pengantara yang hijau.

Kata kunci: Biopestisid; cecair ionik; interaksi pelarut-bahan larut; pelarut eutektik dalam; pengekstrakan

\section{INTRODUCTION}

The extraction process is a research loop backbone prior to purification and bio-active constituent's characterization (Sasidharan et al. 2011). It involves several important processing parameters which influence the efficacy of the extracts. In fact, a conventional extraction process uses a variety of organic solvents which include from highly polar to nonpolar solvents. However, organic solvents are mostly volatile, toxic and flammable leading to several human risks, safety issues and environmental problems (Carson 2002). Taking all this into consideration, there have been several studies on the exploration of ionic liquids (ILs) and deep eutectic solvent (DES) compatibility as green solvents for phytochemicals extraction.

Ionic liquids (ILs) are an organic salt in the liquid state under ambient temperature comprises of normally charge-stabilized organic cation paired with either organic or inorganic anions (Freemantle 2010). While DES is a mixture of two naturally occurring components namely hydrogen bond acceptor (HBA) such as quaternary ammonium halide salts, phosphonium halide salts and metal chloride and hydrogen bond donor (HBD) such as carboxylic acids, alcohols, amides, carbohydrates and metal chloride which associated to each other through hydrogen bond interaction (Abott et al. 2004, 2003). Both solvents display a wide range of unique properties such as high thermal stability, non-flammability, insignificant vapor pressure and low chemical reactivity besides their fine tunable density, viscosity, polarity and miscibility with other common solvents (Bogdanov et al. 2010). For that reason, the usage of selected ILs and DES to aid the phytochemicals extraction process is considered as a great choice in increasing a significant amount of bioactive constituents, while at the same time, reducing 
the use of toxic and volatile organic solvents for a greener environment.

The previous study on rotenone extraction, showed the potential of binary solvent ionic liquid (ILs), 1-butyl-3methylimidazolium trifluoromethanesulfonate, [BMIM]OTf with acetone and alcohol-based DES with acetonitrile as an extractant medium due to higher yield $(\%, \mathrm{w} / \mathrm{w})$ of rotenone extracted of $0.84 \pm 0.05 \%$ and $0.84 \pm 0.02 \%(\mathrm{w} / \mathrm{w})$, respectively (Othman et al. 2015). However, in order to understand what actually lead to both solvent efficiency in extracting high yield rotenone compound, this study aimed to identify the solvent-solute interaction between the selected solvent systems with rotenone compound.

\section{MATERIALS AND METHODS}

\section{CHEMICALS AND MATERIALS}

Choline chloride, $\mathrm{ChCl}(\mathrm{Mw}=139.62$ gmol-1, 99\% $(\mathrm{w} / \mathrm{w})), 1,4-$ butanediol $(\mathrm{Mw}=90.12 \mathrm{gmol}-1,98 \%$ $(\mathrm{w} / \mathrm{w})$, standard rotenone $95 \%(\mathrm{w} / \mathrm{w})$ and 1-butyl-3methylimidazolium trifluoromethanesulfonate [BMIM] OTf $98 \%(\mathrm{v} / \mathrm{v})$ were purchased from Sigma-Aldrich ${ }^{\circledR}$, while acetonitrile 99\% (v/v) and acetone 95\% (v/v) were obtained from Merck.

SOLUTE-SOLVENT INTERACTION STUDY ALCOHOL-BASED DEEP EUTECTIC SOLVENT

Alcohol deep eutectic solvent (DES) was prepared by mixing choline chloride, $\mathrm{ChCl}$ (98\% in purity) with 1 , 4-butanediol (99\% in purity) at a mol ratio of $1 / 5$ according to $\mathrm{Bi}$ et al. (2013). The mixture was continuously stirred at $80^{\circ} \mathrm{C}$ until a homogeneous mixture was obtained. The solution was then kept in Scott bottle once cooled down. The structure elucidation of DES was analyzed using FTIR, ${ }^{1} \mathrm{H}$ NMR and ${ }^{13} \mathrm{C}$ NMR.

\section{BINARY SOLVENT SYSTEMS}

The binary solvent system comprises of two different solvent systems which are DES: acetonitrile and 1-butyl3-methylimidazolium trifluoromethanesulfonate, [BMIM] OTf: acetone. The binary solvent systems were prepared by mixing respective DES and IL with an organic solvent (acetone, acetonitrile) at a ratio of 2:8 (Othman et al. 2017) until homogenous mixture obtained.

\section{SOLVENT-SOLUTE MIXTURE}

The solute-solvent mixture was prepared by mixing standard rotenone with [BMIM] OTf and alcohol-based DES binary solvent systems at a solvent to solid ratio of $10 \mathrm{~mL} / \mathrm{g}$. The mixture was mixed homogeneously using vortex. Once homogenized, the mixture was diluted with a deuterated solvent prior to nuclear magnetic resonance (NMR) analysis. $500 \mu \mathrm{L}$ of deuterated chloroform $\left(\mathrm{CDCl}_{3}\right)$ and deuterated methanol $\left(\mathrm{CD}_{3} \mathrm{OD}\right)$ was added to the prepared mixture and transfer into NMR tube. The prepared samples were then examined through one dimension (1D) and two dimensions (2D) NMR (NOESY, HMBC).

\section{RESULTS AND DISCUSSION}

\section{ELUCIDATION OF ROTENONE}

Structure elucidation of standard rotenone 95\% (Sigma Aldrich) was analyzed using FTIR and 1D-NMR. FTIR spectrum shows the presence of aromatic $\mathrm{CH}$ stretching, aromatic $\mathrm{sp}^{2} \mathrm{C}$ - stretching, methyl and methine group at wavenumber $3081,2976,2939$ and $2814 \mathrm{~cm}^{-1}$, respectively (Figure 1). General wavenumber for carbonyl $(\mathrm{C}=\mathrm{O})$ of ketone was $1705-1725 \mathrm{~cm}^{-1}$, while alkene $(\mathrm{C}=\mathrm{C})$ group stretching were $1620-1680 \mathrm{~cm}^{-1}$. However lower wavenumber of carbonyl $\left(1670 \mathrm{~cm}^{-1}\right)$ and alkene (1596 $\mathrm{cm}^{-1}$ ) stretching observed. This confirms carbonyl conjugation in rotenone structure and clarify the rotenone structure. In addition, it can also be observed that the $\mathrm{C}-\mathrm{H}$ and methine bend as well as $\mathrm{C}-\mathrm{O}$ stretching appeared at an average frequency of 1410-1513, 1416 and 1210-1233 $\mathrm{cm}^{-1}$, respectively. Moreover, proton $\left({ }^{1} \mathrm{H}\right)$ and carbon $\left({ }^{13} \mathrm{C}\right)$ chemical shift of standard rotenone (Table 1) showed comparable results to proton and carbon chemical shift of rotenone reported by $\mathrm{Li}$ et al. (2011). Ftir and 1D-NMR results confirmed the structure of rotenone compound.

\section{STRUCTURAL ELUCIDATION OF [BMIM]OTF AND INTERACTION OCCUR BETWEEN [BMIM]OTF AND ROTENONE}

Structure elucidation of [BMIM] OTf and the solvent-solute interaction study between rotenone and [BMIM] analyzed through FTIR, 1D-NMR and 2D-NMR (NOESY, HMBC). Figure 2 shows the FTIR spectrum of [BMIM]OTf and the solute-solvent mixture. By comparing the two spectrums, it can be observed reduction in the intensity of quaternary amine salt peak which forms with triflate $\left[\mathrm{R}_{3} \mathrm{HN}\right]^{+}[\mathrm{OTf}]^{-}$ionic liquid at wavenumber $3536 \mathrm{~cm}^{-1}$ after interaction with standard rotenone. Besides that, the $\mathrm{C}=\mathrm{N}$ peak of imidazolium ring that appears at wavenumber 1635 $\mathrm{cm}^{-1}$ in [BMIM] OTf spectrum also disappear in the solventsolute mixture spectrum. Although the peak for standard rotenone were not significantly visible due to small ratio of standard in the solvent $(10 \mathrm{~mL} / \mathrm{g})$, the intensity reduction and disappearance of peak in the FTIR spectrum shows that there is a probable bonding pairing between [BMIM] OTf cation with rotenone which weakens the interaction of cation with anion in ionic liquid.

This analysis is supported by a one-dimensional NMR $\left({ }^{1} \mathrm{H}\right.$ and ${ }^{13} \mathrm{C}$ ) and two-dimensional NMR (NOESY, HMBC) of the solvent-solute mixture. Table 2 shows a comparative chemical shift data (ppm) analysis of the binary solvent system. ([BMIM]OTf: acetone) with the solvent-solute mixture. Slight changes in proton chemical shift (ppm) to deshield region were observed for H-2 [BMIM] OTf and $\mathrm{H}-1 \mathrm{a}, \mathrm{H}-6 \mathrm{a} \mathrm{H}-12 \mathrm{a}$ rotenone compared to other protons with an increase of $0.02,0.04,0.03$ and $0.05 \mathrm{ppm}$, respectively. 


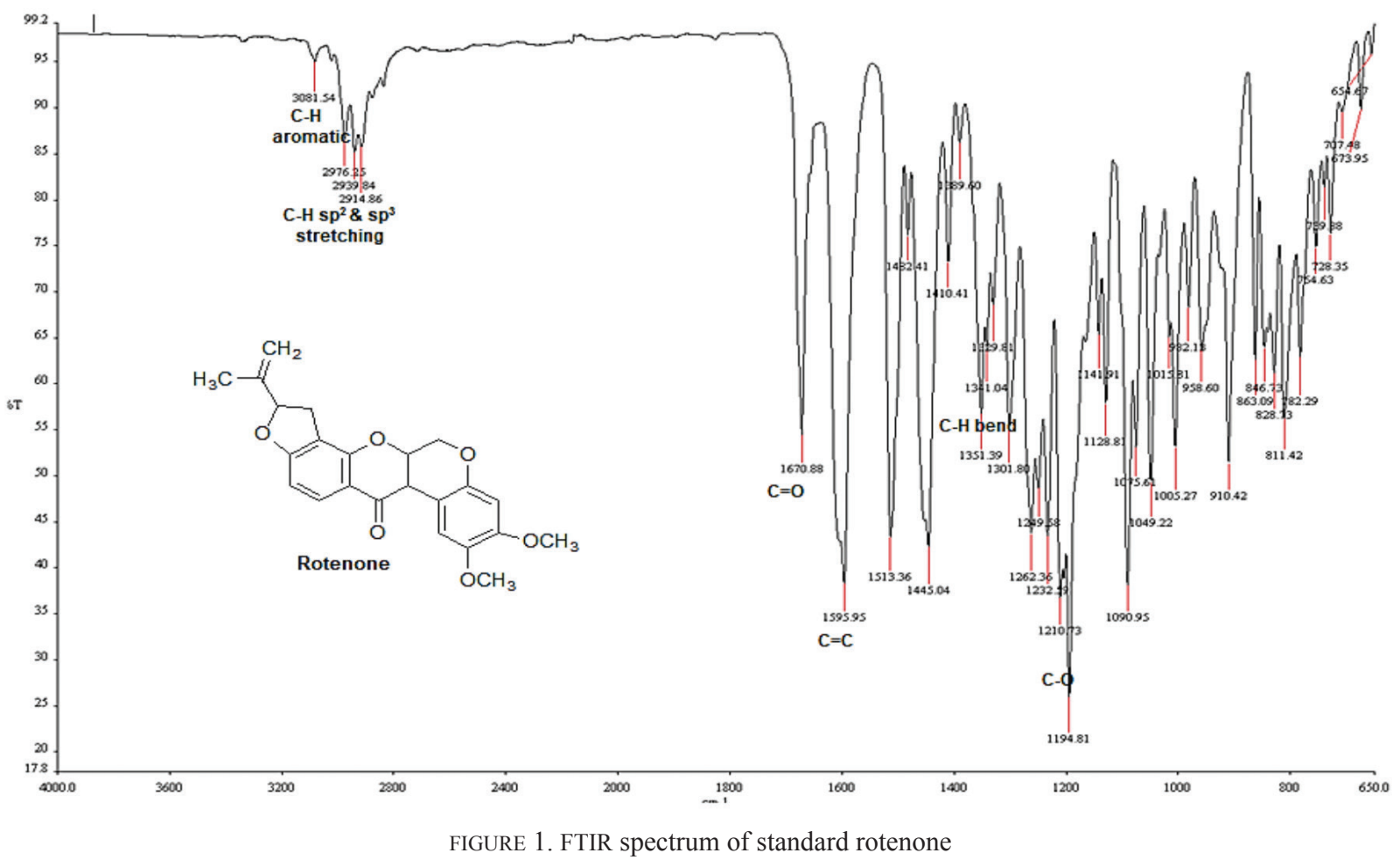

TABLE $1 .{ }^{1} \mathrm{H}$ and ${ }^{13} \mathrm{C}$ chemical shift (ppm) data of standard rotenone and reference $\left(\mathrm{CDCl}_{3}, 400 \mathrm{MHz}\right)$

\begin{tabular}{|c|c|c|c|c|c|}
\hline${ }^{1} \mathrm{H}$ & $\begin{array}{c}\text { Chemical shift (ppm) Std } \\
\text { Rotenone }\end{array}$ & $\begin{array}{c}\text { Chemical shift (ppm) } \\
\text { Reference } \\
\text { (Youzhi et al. 2011) }\end{array}$ & ${ }^{13} \mathrm{C}$ & $\begin{array}{l}\text { Chemical shift } \\
\quad(\mathrm{ppm}) \\
\text { Std Rotenone }\end{array}$ & $\begin{array}{l}\text { Chemical shift } \\
\text { Reference (Youzhi } \\
\text { et al. 2011) }\end{array}$ \\
\hline $\mathrm{CH}_{3}$ & $1.77, \mathrm{~s}, 3 \mathrm{H}$ & $1.77, \mathrm{~s}, 3 \mathrm{H}$ & $\mathrm{CH}_{3}(3 ")$ & 17.21 & 16.8 \\
\hline $\mathrm{OCH}_{3}(8)$ & $3.80, \mathrm{~s}, 3 \mathrm{H}$ & $3.81, \mathrm{~s}, 3 \mathrm{H}$ & $\mathrm{C}-2 "$ & 143.12 & 143.2 \\
\hline $\mathrm{OCH}_{3}(9)$ & $3.77, \mathrm{~s}, 3 \mathrm{H}$ & $3.77, \mathrm{~s}, 3 \mathrm{H}$ & $\begin{array}{l}=\mathrm{C} \mathrm{H}_{2} \\
(1 ”)\end{array}$ & 112.63 & 112.4 \\
\hline$=\mathrm{CH}_{2}(1 ")$ & $5.07,4.93, \mathrm{~s}$ & $5.08, \mathrm{~s}, 1 \mathrm{H}$ & $\mathrm{OCH}_{3}(8)$ & 56.39 & 56 \\
\hline $\mathrm{H}-1$ (a) & $2.95, \mathrm{dd}, 15.6 \mathrm{~Hz}, 8.24 \mathrm{~Hz}$ & $2.95, \mathrm{dd}, 15.7 \mathrm{~Hz}, 8.1 \mathrm{~Hz}$ & $\mathrm{OCH}_{3}(9)$ & 55.92 & 55.4 \\
\hline H-1 (b) & $3.32, \mathrm{dd}, 16.04 \mathrm{~Hz}, 10.08 \mathrm{~Hz}$ & $3.31, \mathrm{dd}, 15.6 \mathrm{~Hz}, 9.8 \mathrm{~Hz}$ & $\mathrm{C}=\mathrm{O}$ & 189.00 & 188.9 \\
\hline $\mathrm{H}-2$ & $5.23, \mathrm{t}, 8.94 \mathrm{~Hz}$ & $5.24, \mathrm{t}, 9.30 \mathrm{~Hz}$ & $\mathrm{C}-2$ & 87.91 & 87.1 \\
\hline $\mathrm{H}-4$ & $6.50, \mathrm{~d}, 8.68 \mathrm{~Hz}$ & $6.50, \mathrm{~d}, 8.5 \mathrm{~Hz}$ & $\mathrm{C}-1$ & 31.35 & 30.60 \\
\hline H-5 & $7.84, \mathrm{~d}, 8.68 \mathrm{~Hz}$ & $7.83, \mathrm{~s}, 1 \mathrm{H}$ & $C-3 a$ & 167.44 & 166.7 \\
\hline $\mathrm{H}-7$ & $6.77, \mathrm{~s}, 1 \mathrm{H}$ & $6.77, \mathrm{~s}, 1 \mathrm{H}$ & C-4 & 104.91 & 104.3 \\
\hline $\mathrm{H}-10$ & $6.45, \mathrm{~s}, 1 \mathrm{H}$ & $6.45, \mathrm{~s}, 1 \mathrm{H}$ & C-5 & 130.05 & 129.2 \\
\hline $\mathrm{H}-12$ (d) & $4.61, \mathrm{dd}, 11.88 \mathrm{~Hz}, 3.2 \mathrm{~Hz}$ & $4.60, \mathrm{dd}, 12.1 \mathrm{~Hz}, 3.0 \mathrm{~Hz}$ & C-5a & 113.42 & 113.3 \\
\hline H-12 (c) & $4.18, \mathrm{~d}, 12.36 \mathrm{~Hz}$ & $4.17, \mathrm{~d}, 12.1 \mathrm{~Hz}$ & C-6a & 44.67 & 43.5 \\
\hline H-6 (a) & $3.83, \mathrm{~d}, 4.12 \mathrm{~Hz}$ & $3.84, \mathrm{~d}, 3.0 \mathrm{~Hz}$ & $C-6 b$ & 104.96 & 105.2 \\
\hline H-12 (a) & $4.93, \mathrm{~m}$ & $4.93, \mathrm{~s}$ & $\begin{array}{l}\text { C-7 } \\
\text { C-8 } \\
\text { C-9 } \\
\text { C-10 } \\
\text { C-10a } \\
\text { C-12 } \\
\text { C-12a } \\
\text { C-13a } \\
\text { C-13b }\end{array}$ & $\begin{array}{l}110.44 \\
143.94 \\
149.56 \\
100.99 \\
147.46 \\
66.35 \\
72.30 \\
158.02 \\
113.04\end{array}$ & $\begin{array}{l}110.7 \\
143.3 \\
149.5 \\
101.4 \\
147.7 \\
65.8 \\
71.7 \\
157.8 \\
113\end{array}$ \\
\hline
\end{tabular}




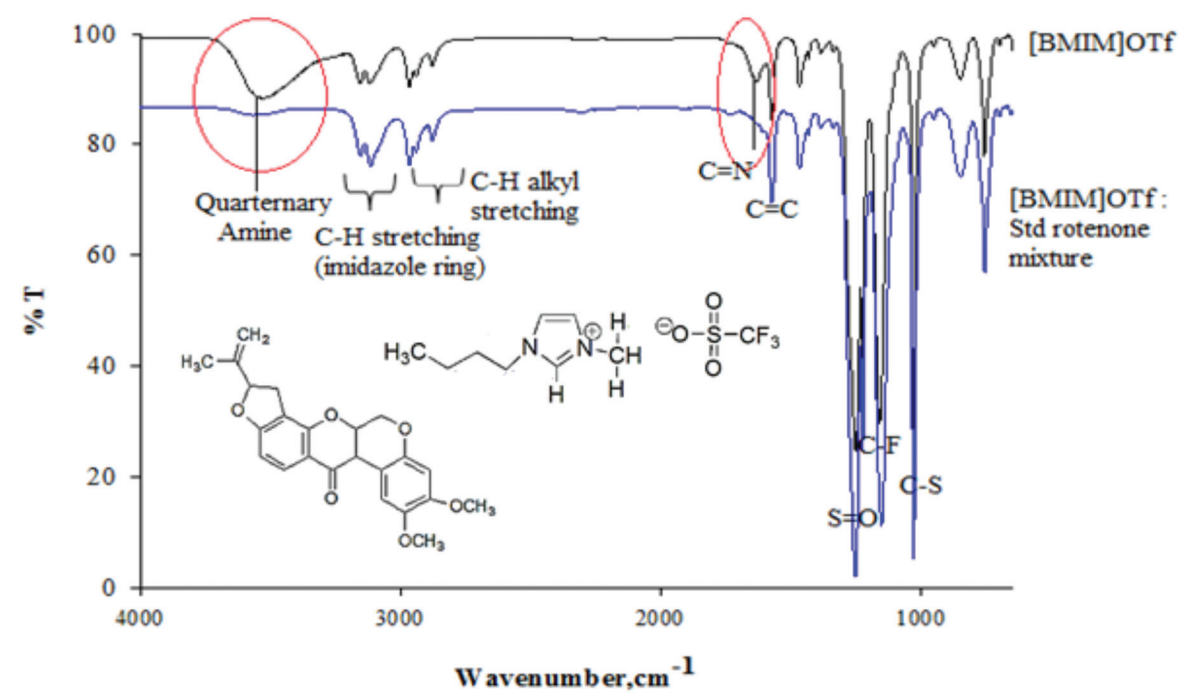

FIGURE 2. Comparison between FTIR spectrum of [BMIM]OTf (black) and FTIR spectrum of [BMIM]OTf: Std rotenone mixture (blue)

TABLE 2. Comparative chemical shift (ppm) data analysis of binary solvent system ([BMIM]OTf: acetone) with chemical shift (ppm) data of solvent-solute mixture $\left(\mathrm{CDCl}_{3}, 400 \mathrm{MHz}\right)$

\begin{tabular}{|c|c|c|c|c|c|c|}
\hline & ${ }^{1} \mathrm{H}$ & $\begin{array}{l}\text { Chemical shift (ppm), } \\
\text { Mixture }\end{array}$ & $\begin{array}{l}\text { Chemical shift } \\
(\mathrm{ppm}) \text { Solvent }\end{array}$ & ${ }^{13} \mathrm{C}$ & $\begin{array}{c}\text { Chemical } \\
\text { shift (ppm) } \\
\text { (DEPT135), } \\
\text { Mixture }\end{array}$ & $\begin{array}{c}\text { Chemical } \\
\text { shift (ppm) } \\
\text { Solvent }\end{array}$ \\
\hline \multirow{10}{*}{$\begin{array}{l}\text { [BMIM] } \\
\text { OTf }\end{array}$} & $\mathrm{CH}_{3}(3 \mathrm{a})$ & $4.00, \mathrm{~s}, 3 \mathrm{H}$ & $3.99, \mathrm{~s}, 3 \mathrm{H}$ & $\mathrm{CH}_{3}(3 \mathrm{a})$ & 36.26 & 36.27 \\
\hline & $\mathrm{CH}_{3}(4 ")$ & $0.96, \mathrm{t}, 7.32 \mathrm{~Hz}$ & $0.96, \mathrm{t}, 7.32 \mathrm{~Hz}$ & $\mathrm{CH}_{3}(4 ")$ & 13.23 & 13.24 \\
\hline & $\mathrm{CH}_{2}(3 ")$ & 1.37, sext, $2 \mathrm{H}$ & 1.37, sext, $2 \mathrm{H}$ & $\mathrm{C}-3 "$ & 19.31 & 19.31 \\
\hline & $\mathrm{CH}_{2}(2 ”)$ & $1.88, \mathrm{q}, 2 \mathrm{H}$ & $1.87, \mathrm{q}, 2 \mathrm{H}$ & C-2" & 31.91 & 31.91 \\
\hline & $\mathrm{CH}_{2}(1 ")$ & $4.23, \mathrm{t}, 7.32 \mathrm{~Hz}$ & $4.23, \mathrm{t}, 7.34 \mathrm{~Hz}$ & C-1" & 49.76 & 49.77 \\
\hline & $\mathrm{H}-4$ & $7.47, \mathrm{~m}$ & $7.46, \mathrm{~d}, 1.84 \mathrm{~Hz}$ & $\mathrm{C}-2$ & 136.64 & 136.63 \\
\hline & $\mathrm{H}-5$ & $7.46, \mathrm{~m}$ & $7.45, \mathrm{~d}, 1.84 \mathrm{~Hz}$ & C-4 & 123.73 & 123.71 \\
\hline & $\mathrm{H}-2$ & $9.13, \mathrm{~s}, 1 \mathrm{H}$ & $9.11, \mathrm{~s}, 1 \mathrm{H}$ & C-5 & 122.33 & 122.29 \\
\hline & $\mathrm{CH}_{3}$ acetone & $2.17, \mathrm{~s}, 6 \mathrm{H}$ & $2.17, \mathrm{~s}, 6 \mathrm{H}$ & CF3 & - & 119.08 \\
\hline & & & & $\mathrm{CH}_{3}$ acetone & 30.82 & 30.73 \\
\hline \multirow{23}{*}{ Rotenone } & $\mathrm{CH}_{3}$ & $1.77, \mathrm{~s}, 3 \mathrm{H}$ & $1.77, \mathrm{~s}, 3 \mathrm{H}$ & $\mathrm{CH}_{3}(3 ”)$ & 17.03 & 17.21 \\
\hline & $\mathrm{OCH}_{3}(8)$ & $3.81, \mathrm{~s}, 3 \mathrm{H}$ & $3.80, \mathrm{~s}, 3 \mathrm{H}$ & $\mathrm{C}-2 "$ & - & 143.12 \\
\hline & $\mathrm{OCH}_{3}(9)$ & $3.76, \mathrm{~s}, 3 \mathrm{H}$ & $3.77, \mathrm{~s}, 3 \mathrm{H}$ & $=\mathrm{CH}_{2}(1 ")$ & 112.46 & 112.63 \\
\hline & $=\mathrm{CH}_{2}(1 ")$ & $5.08,4.94, \mathrm{~s}$ & $5.07,4.93, \mathrm{~s}$ & $\mathrm{OCH}_{3}(8)$ & 56.30 & 56.39 \\
\hline & $\mathrm{H}-1$ (a) & $2.99, \mathrm{dd}, 8.24 \mathrm{~Hz}$ & $2.95, \mathrm{dd}, 15.6 \mathrm{~Hz}, 8.24 \mathrm{~Hz}$ & $\mathrm{OCH}_{3}^{3}(9)$ & 55.79 & 55.92 \\
\hline & $\mathrm{H}-1$ (b) & $3.30, \mathrm{dd}, 10.08 \mathrm{~Hz}, 16.04 \mathrm{~Hz}$ & $3.32, \mathrm{dd}, 16.04 \mathrm{~Hz}, 10.08 \mathrm{~Hz}$ & $\mathrm{C}=\mathrm{O}$ & 206.95 & 189.00 \\
\hline & $\mathrm{H}-2$ & $5.25, \mathrm{t}$ & $5.23, \mathrm{t}, 8.94 \mathrm{~Hz}$ & $\mathrm{C}-2$ & 87.81 & 87.91 \\
\hline & $\mathrm{H}-4$ & $6.50, \mathrm{~d}, 8.24 \mathrm{~Hz}$ & $6.50, \mathrm{~d}, 8.68 \mathrm{~Hz}$ & $\mathrm{C}-1$ & 31.19 & 31.35 \\
\hline & H-5 & $7.83, \mathrm{~d}, 8.72 \mathrm{~Hz}$ & $7.84, \mathrm{~d}, 8.68 \mathrm{~Hz}$ & $\mathrm{C}-3 \mathrm{a}$ & - & 167.44 \\
\hline & $\mathrm{H}-7$ & $6.76, \mathrm{~s}, 1 \mathrm{H}$ & $6.77, \mathrm{~s}, 1 \mathrm{H}$ & $\mathrm{C}-4$ & 104.73 & 104.91 \\
\hline & $\mathrm{H}-10$ & $6.46, \mathrm{~s}, 1 \mathrm{H}$ & $6.45, \mathrm{~s}, 1 \mathrm{H}$ & C-5 & 129.83 & 130.05 \\
\hline & $\mathrm{H}-12$ (d) & $4.62, \mathrm{dd}, 12.36 \mathrm{~Hz}, 3.2 \mathrm{~Hz}$ & $4.61, \mathrm{dd}, 11.88 \mathrm{~Hz}, 3.2 \mathrm{~Hz}$ & $C-5 a$ & - & 113.42 \\
\hline & $\mathrm{H}-12$ (c) & 4.20 & $4.18, \mathrm{~d}, 12.36 \mathrm{~Hz}$ & C-6a & 44.50 & 44.67 \\
\hline & H-6 (a) & $3.86, \mathrm{~d}, 3.68 \mathrm{~Hz}$ & $3.83, \mathrm{~d}, 4.12 \mathrm{~Hz}$ & $\mathrm{C}-6 \mathrm{~b}$ & - & 104.96 \\
\hline & H-12 (a) & $4.98, \mathrm{~m}$ & $4.93, \mathrm{~m}$ & $\mathrm{C}-7$ & 110.50 & 110.44 \\
\hline & & & & C-8 & - & 143.94 \\
\hline & & & & C-9 & - & 149.56 \\
\hline & & & & $\mathrm{C}-10$ & 100.94 & 100.99 \\
\hline & & & & C-10a & - & 147.46 \\
\hline & & & & $\mathrm{C}-12$ & 66.25 & 66.35 \\
\hline & & & & $C-12 a$ & 72.19 & 72.30 \\
\hline & & & & C-13a & - & 158.02 \\
\hline & & & & C-13b & - & 113.04 \\
\hline
\end{tabular}


While for carbon chemical shifts (ppm), it was found that almost all the carbon has a different chemical shift in the mixture. C-4 and C-5 chemical shift (ppm) of [BMIM] slightly deshield with 0.02 and 0.04 ppm increase, while rotenone carbonyl carbon chemical shift up to $17.95 \mathrm{ppm}$ increase. Change in chemical shift to deshield region might due to interaction occurrence around acidic proton $(\mathrm{H}-2)$ of [BMIM] cation with rotenone compound.

This interaction was further convinced through NOESY spectrum (Figure 3(a)) which shows how close solute and solvent in space. There was a correlation between 1) $\mathrm{H}-1$ rotenone with proton $\mathrm{CH}_{3}$ (4") [BMIM] OTf, 2) H-1 rotenone with proton $\mathrm{CH}_{2}$ (3") [BMIM] OTf, 3) H-4 and $\mathrm{H}-10$ rotenone with protons $\mathrm{H}-2$ [BMIM] OTf. This correlation show that $\mathrm{H}-1, \mathrm{H}-4$ and $\mathrm{H}-10$ of rotenone were close in space with alkyl $\left(\mathrm{CH}_{3}(4 "), \mathrm{CH}_{2}(3 ")\right)$ and acidic proton (H-2) of [BMIM] cation. Meanwhile, the HMBC spectrum (Figure 3(b)) supports through long-distance correlation between $\mathrm{CH}_{3}$ (3") [BMIM] OTf with $=\mathrm{CH}_{2}$ standard rotenone. All of the correlation indicates the probability
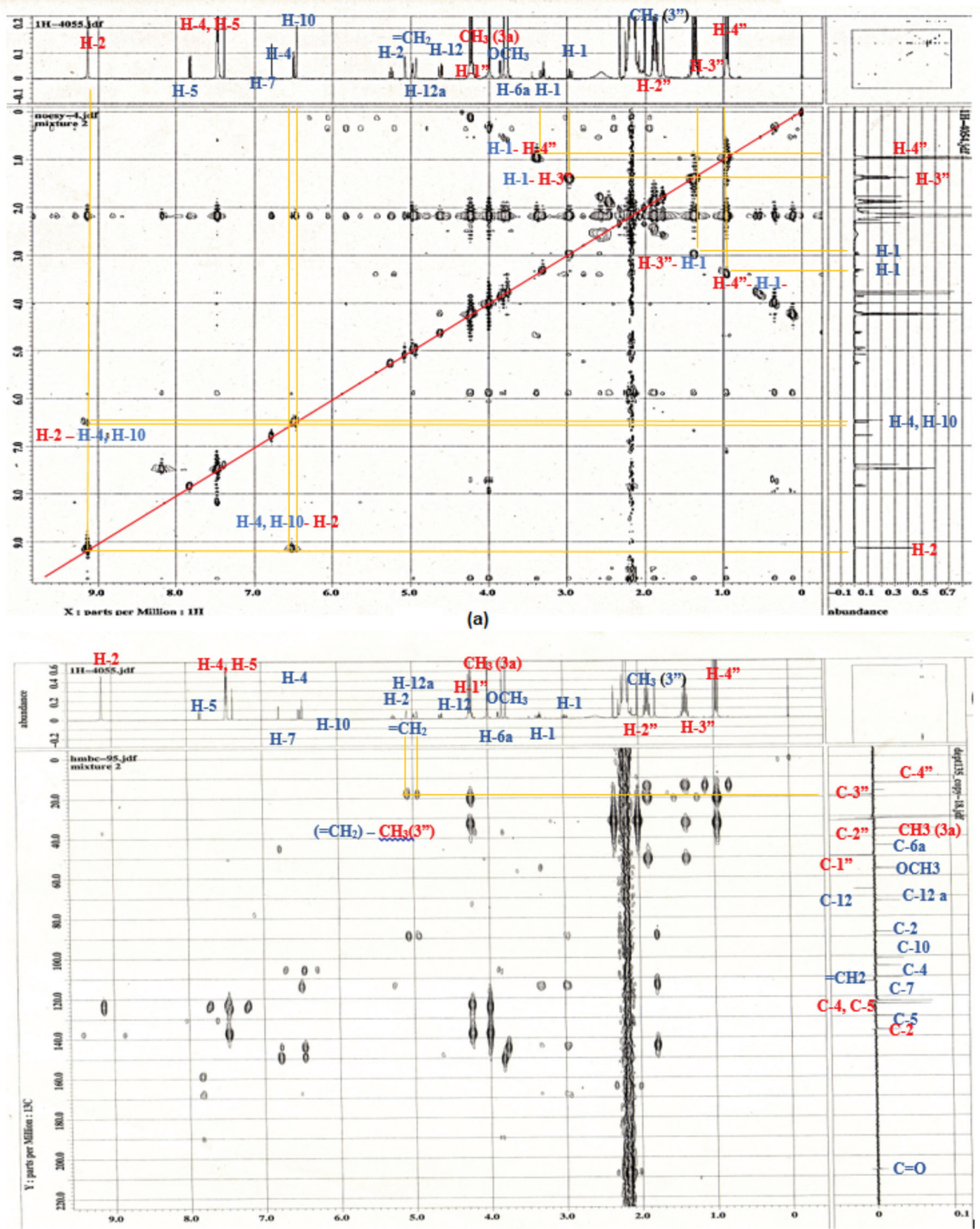

(b)

FIGURE 3. a) NOESY spectrum of mixture [BMIM]OTf: acetone: rotenone and b) HMBC spectrum of mixture [BMIM]OTf: acetone: rotenone. $(*$ Blue $=$ rotenone,$*$ Red $=[$ BMIM $] \mathrm{OTf})$ 
of hydrogen bonding between the acidic hydrogen $(\mathrm{C} 2-\mathrm{H})$ of imidazolium ring with oxygen of rotenone. This bond is likely a contributing factor to the effectiveness of [BMIM] OTf in extracting rotenone compounds in high quantities. Figure 6(a) portrays predicted interaction of ionic liquids, [BMIM]OTf with solute (rotenone standard).

\section{STRUCTURAL ELUCIDATION OF DEEP EUTECTIC SOLVENT (DES) AND INTERACTION OCCUR BETWEEN DES AND ROTENONE}

The prepared DESs with a density of $0.9664 \mathrm{~g} / \mathrm{mL}$ were elucidated using FTIR, ${ }^{1} \mathrm{H}$ NMR and ${ }^{13} \mathrm{C}$ NMR. Based on the FTIR spectrum (Figure 4(a)) it could be observed that the broad $\mathrm{OH}$ bond stretched at $3294 \mathrm{~cm}^{-1}$ of choline chloride, $\mathrm{ChCl}$ and 1, 4-butanediol along with $\mathrm{Sp}^{3} \mathrm{C}-\mathrm{H}$ that reached its stretching peak at 2937 and $2868 \mathrm{~cm}^{-1}$. $\mathrm{CH}_{2}$ and $\mathrm{CH}_{3}$ bending were also observed at 1478 and $1417 \mathrm{~cm}^{-1}$, respectively. Meanwhile, $\mathrm{C}-\mathrm{N}^{+}$symmetric stretching of choline chloride, $\mathrm{ChCl}$ was observed at $748 \mathrm{~cm}^{-1}$. There were several peaks that represented by 1, 4-butanediol. The peaks were $\mathrm{C}-\mathrm{O}-\mathrm{H}$ bond bending (multi-peaks, broad and weak) at a range of 1300-1200 $\mathrm{cm}^{-1}$ and $\mathrm{C}-\mathrm{O}$ bond stretching peak in $1^{\circ}$ alcohol at 1049 $\mathrm{cm}^{-1}$. Choline chloride, $\mathrm{ChCl}$ was used as hydrogen bond acceptor (HBA) while 1, 4-butanediol was used as hydrogen bond donor (HBD). The stretching vibrations of alcohol in both 1,4-butanediol and choline chloride, $\mathrm{ChCl}$ shifted to lower frequencies from $3340 \mathrm{~cm}^{-1}$ in $\mathrm{ChCl}$ (Park et al. 2013) to $3294 \mathrm{~cm}^{-1}$ in 1,4- butanediol DES, which was prepared by mixing two components. It is known that

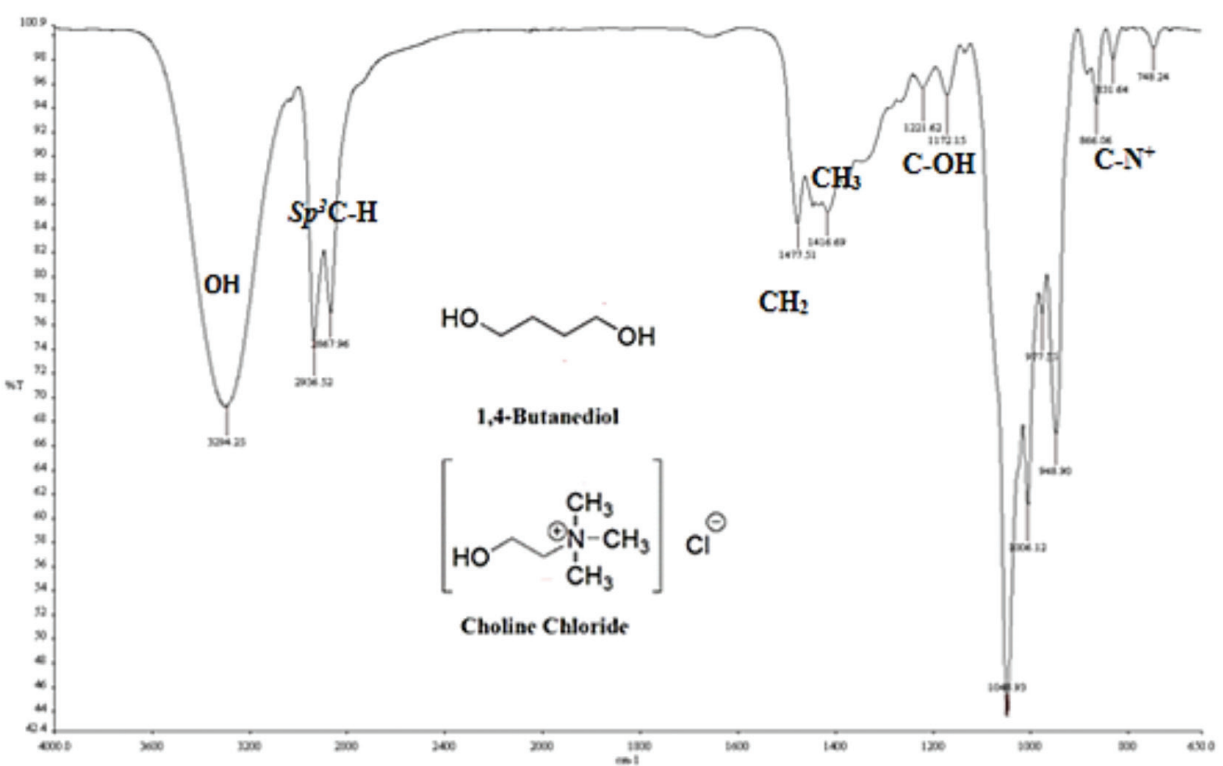

(a)

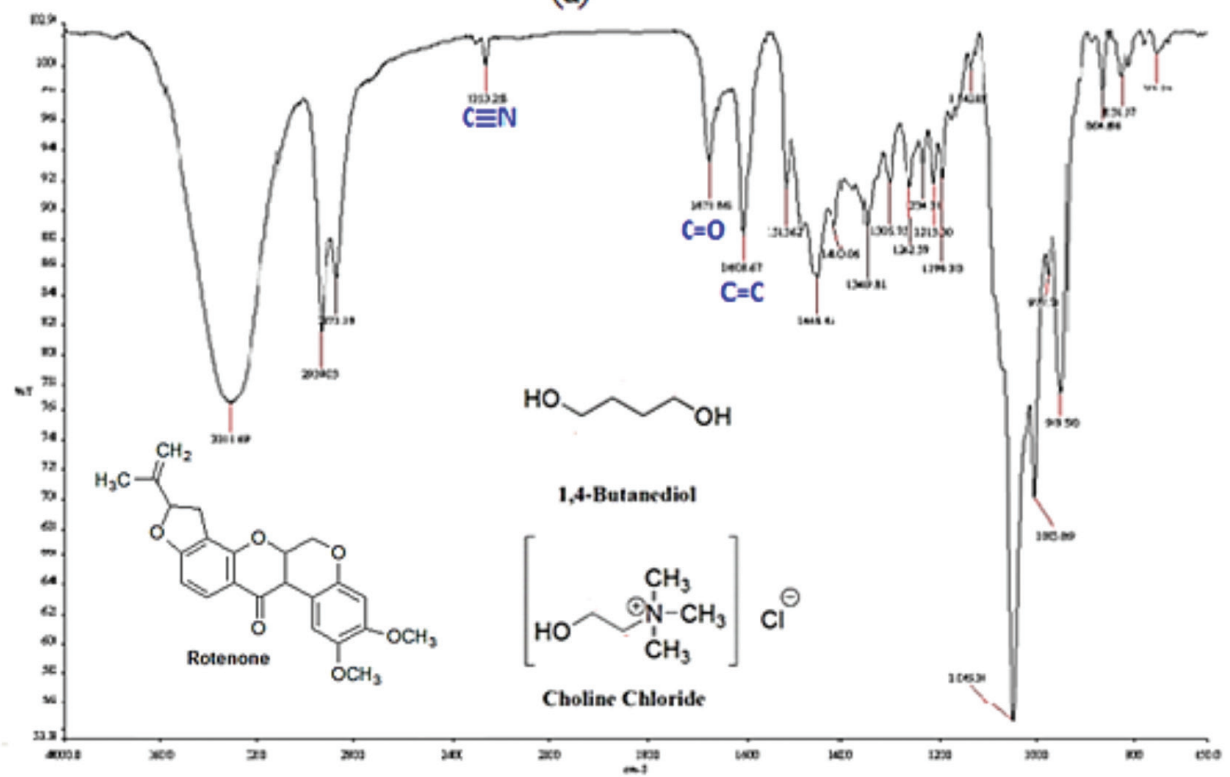

(b)

FIGURE 4. a) FTIR spectrum of DES b) FTIR spectrum of solute-solvent mixture (STD rotenone: DES) 
the existence of the main hydrogen bonding in DES was due to the bonding between $\mathrm{Cl}^{-}$ion in $\mathrm{ChCl}$ and hydrogen donor molecule (Shamsuri \& Daik 2012). Nevertheless, the shift to lower frequencies of hydroxyl stretching vibration could be attributed to the potential hydrogen bond formation through hydroxyl group in $\mathrm{ChCl}$.

The proton nuclear magnetic resonance, ${ }^{1} \mathrm{H}-\mathrm{NMR}$ $(600 \mathrm{MHz})$ spectrum of DES was obtained in deuterated methanol ( $\left.\mathrm{CD}_{3} \mathrm{OD}\right)$. The ${ }^{1} \mathrm{H}-\mathrm{NMR}$ spectrum showed the mixture of choline chloride, $\mathrm{ChCl}$ and 1, 4-butanediol, where the chemical shift for choline chloride, $\mathrm{ChCl}$ appeared at $\delta(\mathrm{ppm}) 3.255(\mathrm{~s}, 9 \mathrm{H}), 3.530-3.592(\mathrm{~m}, 2 \mathrm{H})$ and 4.016-4.041 (m, 2H). While for 1, 4-butanediol, the chemical shift was observed at $\delta(\mathrm{ppm}) 1.591-1.635$ (m, $2 \mathrm{H})$ and 3.583-3.592 (m, 2H).

The carbon-13 nuclear magnetic resonance, ${ }^{13} \mathrm{C}$-NMR $(600 \mathrm{MHz})$ spectrum of DES was also obtained in deuterated methanol ( $\left.\mathrm{CD}_{3} \mathrm{OD}\right)$. The chemical shift for choline chloride, $\mathrm{ChCl}$ appeared at $\delta(\mathrm{ppm}) 53.35\left(\mathrm{CH}_{3}\right)$, 55.71( $\mathrm{CH} 2)$ and $67.62\left(\mathrm{CH}_{2}\right)$. While for 1, 4-butanediol, the chemical shift was observed at $\delta(\mathrm{ppm}) 28.79\left(\mathrm{CH}_{2}\right)$ and $61.49\left(\mathrm{CH}_{2}\right)$. Both ${ }^{1} \mathrm{H}$-NMR and ${ }^{13} \mathrm{C}$-NMR spectrum chemical shifts were comparable to the previous elucidation on DES as according to Dai et al. (2013). Thus, confirm the structure of alcohol-based DES.

FTIR spectroscopy, 1D-NMR and 2D- NMR (NOESY and $\mathrm{HMBC}$ ) were used to determine the location and interaction between a binary solvent, (DES: acetonitrile) with solute (standard rotenone). In FTIR spectra (Figure 4(b)) of the mixture, it can be observed the existence of acetonitrile cyanide peak, $\mathrm{C} \equiv \mathrm{N}$, carbonyl $(\mathrm{C}=\mathrm{O})$ and alkenes $(\mathrm{C}=\mathrm{C})$ of rotenone which previously not observed in DES FTIR spectrum. However, observations only showed the presence of rotenone and acetonitrile without a clear picture of the interaction between the solute (STD rotenone) with alcohol-based deep eutectic solvent (DES).

Therefore, the chemical shift (ppm) data analysis of solute-solvent mixture using a one-dimensional NMR was compared to the chemical shifts (ppm) of binary solvent (DES: acetonitrile) in Table 3 to identify the changes in

TABLE 3. Comparative chemical shift (ppm) data analysis of binary solvent system (DES: acetonitrile) with chemical shift (ppm) data of solvent-solute mixture, $\left(\mathrm{CD}_{3} \mathrm{OD}, 400 \mathrm{MHz}\right)$

\begin{tabular}{|c|c|c|c|c|c|c|}
\hline & ${ }^{1} \mathrm{H}$ & $\begin{array}{l}\text { Chemical shift (ppm) } \\
\text { Mixture }\end{array}$ & $\begin{array}{c}\text { Chemical shift (ppm) } \\
\text { Solvent }\end{array}$ & ${ }^{13} \mathrm{C}$ & $\begin{array}{l}\text { Chemical } \\
\text { shift (ppm) } \\
\text { Mixture }\end{array}$ & $\begin{array}{c}\text { Chemical } \\
\text { shift (ppm) } \\
\text { Solvent }\end{array}$ \\
\hline \multirow{6}{*}{ DES } & $\mathrm{CH}_{3}$ & $3.29, \mathrm{~s}$ & $3.30, \mathrm{~s}, 9 \mathrm{H}$ & $\mathrm{CH}_{3}$ & - & - \\
\hline & $\mathrm{H}-2 " / \mathrm{H}-3 "$ & $1.56, \mathrm{~m}, 2 \mathrm{H}$ & $1.58, \mathrm{~m}, 2 \mathrm{H}$ & $\mathrm{C}-2 " / \mathrm{C}-3 "$ & 28.99 & 29.19 \\
\hline & H-1"/H-4" & $3.55, \mathrm{~m}, 2 \mathrm{H}$ & $3.56, \mathrm{~m}, 2 \mathrm{H}$ & C-1"/ C-4" & 61.59 & 61.84 \\
\hline & $\mathrm{H}-1$ & $4.07, \mathrm{~m}$ & $3.73, \mathrm{~m}, 2 \mathrm{H}$ & $\mathrm{C}-1$ & - & - \\
\hline & $\mathrm{H}-2$ & $3.37, \mathrm{~m}$ & $3.38, \mathrm{t}, 2 \mathrm{H}, 4.8 \mathrm{~Hz}$ & $\mathrm{C}-2$ & - & - \\
\hline & & & & $\mathrm{C} \equiv \mathrm{N}$ & - & 117.30 \\
\hline \multirow{23}{*}{ Rotenone } & $\mathrm{CH}_{3}$ & $1.74, \mathrm{~s}, 3 \mathrm{H}$ & $1.77, \mathrm{~s}, 3 \mathrm{H}$ & $\mathrm{CH}_{3}\left(3^{\prime \prime}\right)$ & 16.08 & 17.21 \\
\hline & $\mathrm{OCH}_{3}(8)$ & $3.75, \mathrm{~s}, 3 \mathrm{H}$ & $3.80, \mathrm{~s}, 3 \mathrm{H}$ & $\mathrm{C}-2 "$ & 143.74 & 143.12 \\
\hline & $\mathrm{OCH}_{3}(9)$ & $3.66 \mathrm{~s}, 3 \mathrm{H}$ & $3.77, \mathrm{~s}, 3 \mathrm{H}$ & $=\mathrm{CH} 2(1 ")$ & 111.69 & 112.63 \\
\hline & $=\mathrm{CH}_{2}(1 ")$ & $5.05,4.92, \mathrm{~s}$ & $5.07,4.93, \mathrm{~s}$ & $\mathrm{OCH}_{3}(8)$ & 55.96 & 56.39 \\
\hline & H-1 (a) & $2.93, \mathrm{dd}, 8.24 \mathrm{~Hz}, 15.56 \mathrm{~Hz}$ & $2.95, \mathrm{dd}, 15.6 \mathrm{~Hz}, 8.24 \mathrm{~Hz}$ & $\mathrm{OCH}_{3}(9)$ & 55.33 & 55.92 \\
\hline & H-1 (b) & $3.27, \mathrm{dd}, 9.60 \mathrm{~Hz}, 15.56 \mathrm{~Hz}$ & $3.32, \mathrm{dd}, 16.04 \mathrm{~Hz}, 10.08 \mathrm{~Hz}$ & $\mathrm{C}=\mathrm{O}$ & 189.83 & 189.00 \\
\hline & $\mathrm{H}-2$ & $5.29, \mathrm{t}, 8.92 \mathrm{~Hz}$ & $5.23, \mathrm{t}, 8.94 \mathrm{~Hz}$ & $\mathrm{C}-2$ & 87.91 & 87.91 \\
\hline & $\mathrm{H}-4$ & $6.50, \mathrm{~d}, 8.24 \mathrm{~Hz}$ & $6.50, \mathrm{~d}, 8.68 \mathrm{~Hz}$ & $\mathrm{C}-1$ & 30.79 & 31.35 \\
\hline & $\mathrm{H}-5$ & $7.78, \mathrm{~d}, 8.24 \mathrm{~Hz}$ & $7.84, \mathrm{~d}, 8.68 \mathrm{~Hz}$ & C-3a & 167.49 & 167.44 \\
\hline & $\mathrm{H}-7$ & $6.70, \mathrm{~s}, 1 \mathrm{H}$ & $6.77, \mathrm{~s}, 1 \mathrm{H}$ & $\mathrm{C}-4$ & 104.49 & 104.91 \\
\hline & $\mathrm{H}-10$ & $6.48, \mathrm{~s}, 1 \mathrm{H}$ & $6.45, \mathrm{~s}, 1 \mathrm{H}$ & C-5 & 129.61 & 130.05 \\
\hline & $\mathrm{H}-12$ (d) & $4.55, \mathrm{dd}, 12.36 \mathrm{~Hz}, 2.76 \mathrm{~Hz}$ & $4.61, \mathrm{dd}, 11.88 \mathrm{~Hz}, 3.2 \mathrm{~Hz}$ & $C-5 a$ & 113.30 & 113.42 \\
\hline & $\mathrm{H}-12$ (c) & $4.20, \mathrm{~d}, 12.4 \mathrm{~Hz}$ & $4.18, \mathrm{~d}, 12.36 \mathrm{~Hz}$ & C-6a & 44.29 & 44.67 \\
\hline & H-6 (a) & $3.86, \mathrm{~d}, 4.12 \mathrm{~Hz}$ & $3.83, \mathrm{~d}, 4.12 \mathrm{~Hz}$ & $C-6 b$ & 111.07 & 104.96 \\
\hline & H-12 (a) & $5.01, \mathrm{~m}$ & $4.93, \mathrm{~m}$ & $\mathrm{C}-7$ & 111.07 & 110.44 \\
\hline & & & & C-8 & 143.74 & $143 . .94$ \\
\hline & & & & C-9 & 149.92 & 149.56 \\
\hline & & & & $\mathrm{C}-10$ & 101.27 & 100.99 \\
\hline & & & & C-10a & 148.05 & 147.46 \\
\hline & & & & C-12 & 66.10 & 66.35 \\
\hline & & & & C-12a & 72.39 & 72.30 \\
\hline & & & & $C-13 a$ & 158.23 & 158.02 \\
\hline & & & & $C-13 b$ & 113.13 & 113.04 \\
\hline
\end{tabular}


the interactions that occur. Based on Table 3, change in proton chemical shift of $\mathrm{H}-1$ DES and $\mathrm{H}-2, \mathrm{H}-10, \mathrm{H}-6 \mathrm{a}$, $\mathrm{H}-12 \mathrm{a}$ rotenone with increment of $0.34,0.06,0.03,0.03$ and $0.08 \mathrm{ppm}$, respectively. Chemical shift (ppm) of carbon in the ${ }^{13} \mathrm{C}$-NMR spectrum also shows the change in chemical shifting for carbon $\mathrm{C}-2$ ", $\mathrm{C}=\mathrm{O}, \mathrm{C}-6 \mathrm{~b}$ and $\mathrm{C}-7$ rotenone with the increment of $0.62,0.83,6.11$ and 0.63 $\mathrm{ppm}$. This observation is supported by NOESY (Figure 5(a)) and HMBC spectrum (Figure 5(b)). Where from NOESY spectrum can be found that there was a correlation between the hydroxyl of choline chloride $(\mathrm{ChCl})$ with hydroxyl of 1,4-butanediol in space to confirm the existence of hydrogen bonds in DES. Whereas, from
HMBC spectrum can be observed a long-range correlation between 1) $\mathrm{CH}_{3}$ (3") rotenone with $\mathrm{C}-2$ " and $\mathrm{C}-3$ " 1,4-butanediol, 2) $\mathrm{OCH}_{3}$ rotenone with $\mathrm{C}-1$ " and $\mathrm{C}-4$ " 1,4-butanediol. The correlation does not show a clear interaction between the DES with a standard rotenone. In which this correlation only shows interactions likely to occur between the methyl group and methoxy group of rotenone with hydroxyl 1,4-butanediol. This is likely due to the presence of various molecules in a system with a different ratio of each molecule. Therefore, it is difficult to determine the bond that exists between the solvent and solute. Figure 6(b) shows the correlation that exists between DES and the solute (rotenone).

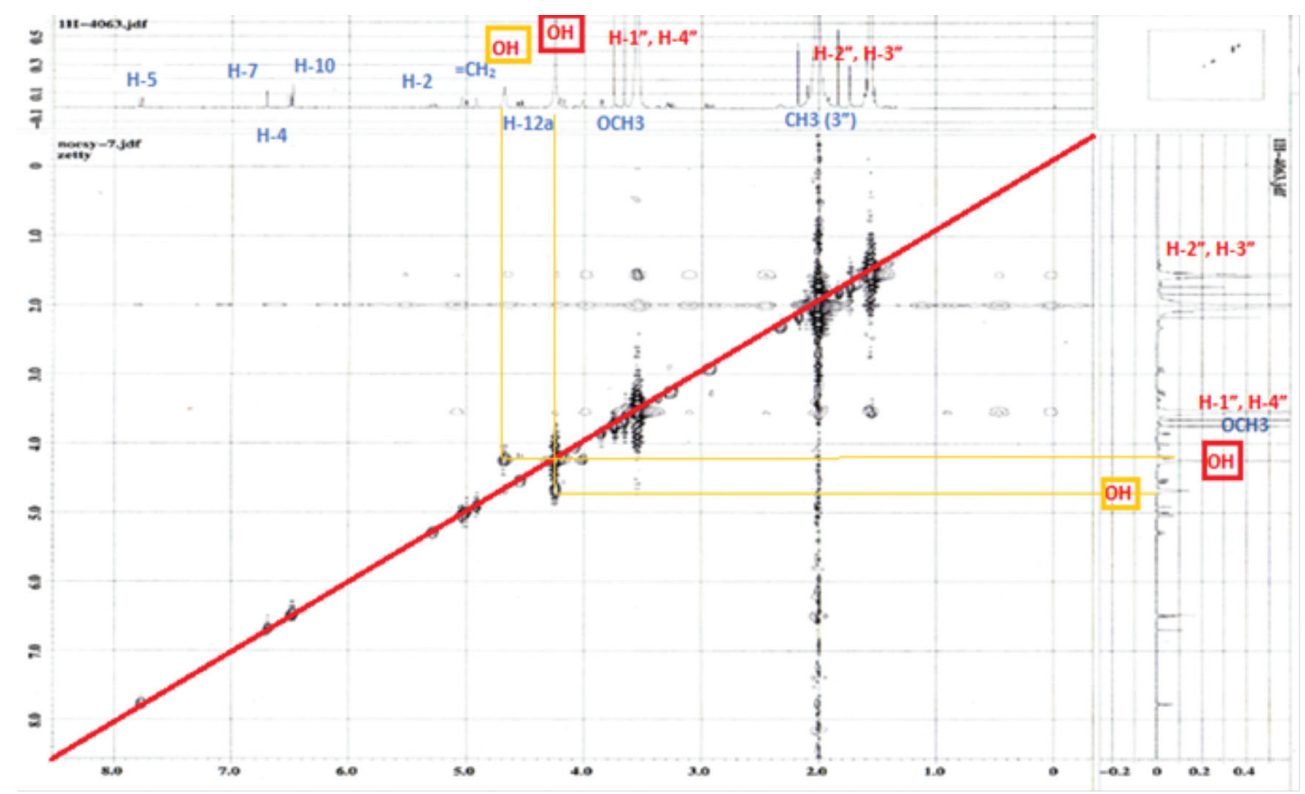

(a)

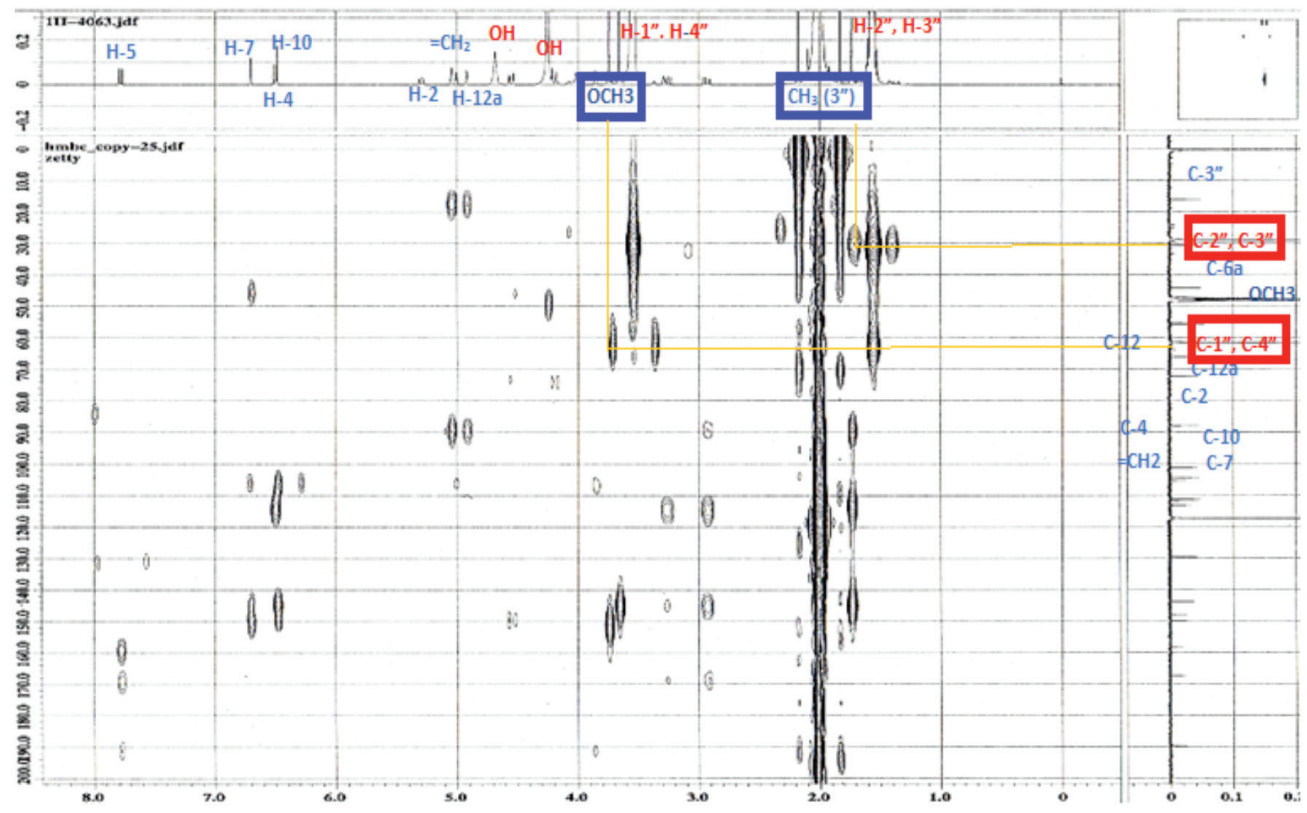

(b)

FIGURE 5. a) NOESY spectrum of mixture (DES: acetonitrile: rotenone) and b) HMBC spectrum of mixture $($ DES: acetonitrile: rotenone $)(*$ Blue $=$ rotenone, $*$ Red $=$ DES $)$ 


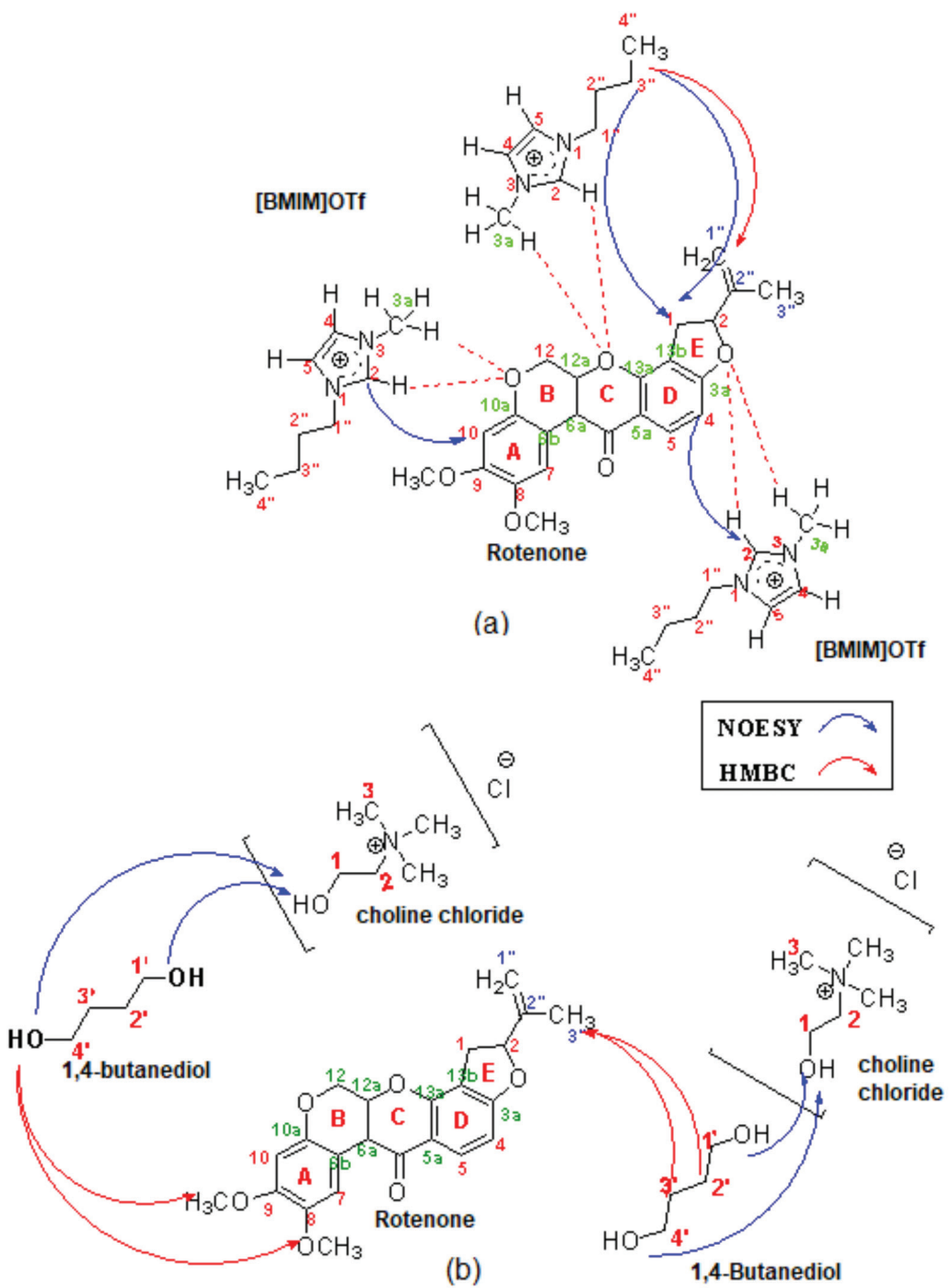

FIGURE 6. Simulation on the interaction between solute (STD rotenone) with (a)[BMIM]OTf (b) DES (NOESY dan HMBC)

\section{CONCLUSION}

In conclusion, both binary solvent systems of [BMIM] OTf and alcohol-based DES have a great potential as an extraction medium compares to volatile organic solvents in extracting optimum rotenone extract. This was due to the presence of probable hydrogen bonding in [BMIM] OTf: rotenone, solvent-solute mixture. Meanwhile for DES interaction with rotenone, there is an interaction between the methyl and methoxy group of rotenone with the hydroxyl group of 1,4-butanediol. All of this interaction makes it possible for both solvent systems to extract more rotenone yield compared to conventional organic solvents. In addition to its extracting potential, DES and [BMIM]OTf also overcoming the drawback of conventional organic solvent enhancing their green properties. Therefore, both DES and [BMIM] OTf application as extraction medium is considered a wise approach to conserve the environment while optimizing the extraction yield of the bioactive compound.

\section{ACKNOWLEDGEMENTS}

The authors would like to thank the Ministry of Higher Education (MOE), Malaysia, for providing financial support to this project (FRGS/2/2013/TK04/UKM/03/1, GUP-2017-014 and GGPM-2013-078) and Center of Research and Instrumentation Management (CRIM), Universiti Kebangsaan Malaysia for the facilities on the instrumentation and analysis.

\section{REFERENCES}

Abbott, A.P., Capper, G., Davies, D.L., Rasheed, R.K. \& Tambyrajah, V. 2003. Novel solvent properties of choline chloride/urea mixtures. Chemical Communications (1): 70-71.

Abbott, A.P., Boothby, D., Capper, G., Davies, D.L. \& Rasheed, R.K. 2004. Deep eutectic solvents formed between choline chloride and carboxylic acids: Versatile alternatives to ionic liquids. Journal of the American Chemical Society 126(29): 9142-9147. 
Bi, W., Tian, M. \& Row, K.H. 2013. Evaluation of alcohol-based deep eutectic solvent in extraction and determination of flavonoids with response surface methodology optimization. Journal of Chromatography A 1285: 22-30.

Bogdanov, M.G., Petkova, D., Hristeva, S., Svinyarov, I. \& Kantlehner, W. 2010. New guanidinium-based room temperature ILs substituent and anion effect on density and solubility in water. Zeitschrift f'ur Naturforschung B 65(1): 37-48.

Carson, P.A. 2002. Hazardous Chemicals Handbook. 2nd ed. Boston: Butterworth- Heinemann.

Dai, Y., Van, S.J., Witkamp, G.J., Verpoorte, R. \& Choi, Y.H. 2013. Natural deep eutectic solvents as new potential media for green technology. Analytica Chimica Acta 766: 61-68.

Freemantle, M. 2010. An Introduction to Ionic Liquid. Cambridge: Royal Society of Chemistr.

Li, Y.Z., Li, G.H., Wei, X.Y., Liu,Z.H. \& Xu, H.H. 2011. Isolation and identification of insecticidal compounds from Tephrosia purpurea (Fabaceae) bark and their insecticidal activity. Acta Entomologica Sinica 54(12): 1368-1376.

Othman, Z.S., Hassan, N.H. \& Zubairi, S.I. 2017. Response surface optimization of rotenone using natural alcohol-based deep eutectic solvent as additive in the extraction medium cocktail. Journal of Chemistry 2017: 1-10.

Othman, Z.S., Hassan, N.H. \& Zubairi, S.I. 2015. Alcohol baseddeep eutectic solvent (DES) as an alternative green additive to increase rotenone yield. In AIP Conference Proceedings 1678(1): 050004.

Park, J.H., Oh, K.W. \& Choi, H.M. 2013. Preparation and characterization of cotton fabrics with antibacterial properties treated by crosslinkable benzophenone derivative in choline chloride-based deep eutectic solvents. Cellulose 20(4): 2101-2114.
Sasidharan, S., Chen, Y., Saravanan, D., Sundram, K.M. \& Yoga, L. 2011. Extraction, isolation and characterization of bioactive compounds from plants extracts. African Journal of Traditional, Complementary and Alternative Medicines 8(1): 1-10.

Shamsuri, A.A. \& Daik, R. 2012. Plasticizing effect of choline chloride/urea eutectic-based ionic liquid on physicochemical properties of agarose films. BioResources 7(4): 4760-4775.

Zetty Shafiqa Othman, Nurul Huda Abd Karim,

Saiful Irwan Zubairi \& Nur Hasyareeda Hassan* School of Chemical Science and Food Technology

Faculty of Science and Technology

Universiti Kebangsaan Malaysia

43600 UKM Bangi, Selangor Darul Ehsan

Malaysia

Mamoru Koketsu

Department of Chemistry and Bimolecular Science Faculty of Engineering

Gifu University, Gifu

Japan

*Corresponding author; email: syareeda@ukm.edu.my

Received: 28 November 2017

Accepted: 4 March 2018 